\title{
BMJ Open Health assets in older age: a systematic review
}

\author{
Yvonne Claire Hornby-Turner, Nancye May Peel, Ruth Eleanor Hubbard
}

To cite: Hornby-Turner YC, Peel NM, Hubbard RE. Health assets in older age: a systematic review. BMJ Open 2017;7:e013226. doi:10.1136/ bmjopen-2016-013226

- Prepublication history and additional material are available. To view, these files please visit the journal online (http://dx.doi.org/ 10.1136/ bmjopen-2016-013226).

Received 28 June 2016 Revised 20 March 2017 Accepted 31 March 2017

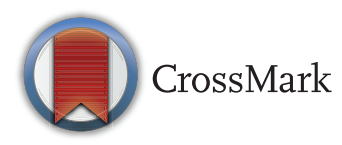

Centre for Research in Geriatric Medicine, Faculty of Medicine, The University of Queensland, Brisbane, Queensland, Australia

Correspondence to

Dr Yvonne Claire Hornby-Turner; y.hornbyturner@uq.edu.au

\begin{abstract}
Background Finding ways to optimise health in older age is key to reducing the impact of population ageing on health and social care systems. A salutogenic approach takes into account an individual's health assets-internal or external strengths or accessible resources which improve and preserve physical, social and mental wellness, independence and quality of life. The aim of this narrative systematic review was to provide a summary and appraisal of the evidence for factors that act as health assets within personal, social, economic and environmental domains.

Methods Systematic searches of databases were conducted for literature published in peer-reviewed journals between January 2000 and November 2016. Selection criteria included community dwelling populations aged 65 years and over and publications written in English. Data on study population, design, measures of health status, factors within the four previously stated domains and results were extracted. Study quality was independently assessed using an appraisal instrument. Results Twenty-three publications, including 78422 participants, from more than 13 different countries were identified for inclusion in this review. There was strong evidence that higher scores of self-rated health, psychological well-being and life satisfaction were associated with better health in older age. Social network and contact with family and friends, and engagement in leisure and social activities were important support mechanisms. Education and financial resources consistently proved to be key economic health assets for older adults.

Conclusions Implementing an asset-based approach to health promotion uncovers the skills, knowledge, connections and potential of the individual and the community. This approach is an ideal opportunity for government health bodies and their partners to respond to the challenges faced by global ageing. Factors are often interdependent and cumulative, suggesting the potential for an instrument to measure the accumulated effect of health assets on health status in older adults.
\end{abstract}

\section{INTRODUCTION}

On a global level, people aged 65 years or older are the fastest growing segment of the population. ${ }^{1}$ While global ageing is perceived as a success, the continued growth of this population will add increasing economic and social demands on all countries. ${ }^{2}$ This demographic shift in global ageing also
Strengths and limitations of this study

- This review has evaluated an extensive range of health assets, highlighting the evidence for factors that positively influence health in older age.

- Of the studies identified for inclusion in this review, methodological differences in study design, followup periods, population samples and the way health assets and health status were measured precluded the pooling of results for meta-analysis.

- The cross-sectional designs of the majority of studies did not allow a cause-effect relationship to be examined between health asset indicators and subsequent health in older age.

entails fundamental social, economic and development challenges and opportunities, not the least of which is the increasing priority to meet the needs of older persons while enabling them to have longer, healthier and more productive lives. ${ }^{3}$ Identifying ways to enhance health and well-being in older age is key to reducing the impact of global ageing, and is therefore a fundamental issue for policy makers. ${ }^{1}$

Based on the WHO definition, health in older age is described as a life course process of optimising opportunities for improving and preserving physical, social and mental wellness, independence, quality of life and enhancing successful transitions. ${ }^{2}{ }^{4}$ This holistic definition recognises that health is multifactorial, spanning across the various domains of well-being. Hence, factors that influence health are complex and wideranging.

In 2002, WHO published the Active ageing: a policy framework. ${ }^{2}$ This framework identifies six key domains of active ageing: economic, behavioural, personal, social, health and social services and the physical environment. ${ }^{2}$ This framework highlights the need for quality evidence to support appropriate policies and programmes across all domains to promote health in older age.

Historically, approaches to the promotion of health have been based on an 'illness' model. The focus is mainly on risk factors 
for disease 'health deficits', rather than those associated with improving health status. While the presence of risk factors increases the likelihood of poor health, their absence does not necessarily increase the likelihood of good health. This approach of identifying risk factors for disease is essential for understanding specific needs and priorities; however, it tends to define individuals in negative terms and may overlook important positive factors which improve public health. ${ }^{5}$

In contrast, a 'wellness' model accentuates a salutogenic approach, concerned with identifying protective factors, 'health assets', to support health and well-being, rather than those that cause disease. ${ }^{6}$ 'Health assets' are defined as an individual's internal or external strengths or accessible resources which enhance ability to optimise health. ${ }^{578}$ Identifying 'health assets' that positively influence or are protective of health in older age will support the design of effective policies and programmes for the promotion of health in older age.

Previous reviews in this research area have examined the concept of health assets in a healthcare context. ${ }^{79}$ Other similar systematic reviews include Peel $e t a l,{ }^{10}$ who identified a broad range of behavioural predictors, and Depp and Jeste, ${ }^{11}$ who examined demographic, psychosocial and biomedical correlates of successful ageing. To our knowledge, however, no other review has provided an overview of 'health assets' or positive health determinants, with a focus on personal, social, economic and environmental predictors of positive health in older age in community-dwelling adults.

The aim of this review was to conduct a narrative summary and appraisal of evidence, published from the year 2000 onwards, for factors that have potential to act as health assets and promote health in older age. Based on the WHO active ageing policy framework, factors within the personal, environmental, economic and social domains were selected with a focus on only those that are protective of health in older age and potentially amenable to change through policy or intervention. Behavioural and lifestyle factors were excluded from this review as they have been the subject of a previous systematic review. ${ }^{10}$

\section{METHODS}

\section{Literature search}

In October 2014, a systematic search of databases (PubMed, Medline, Embase, Cumulative index to nursing and allied health literature and PsycNet) for literature was undertaken to address the study question 'What health assets positively influence health in older age?' Furthermore, additional articles were identified by manually reviewing the reference lists of included papers. An updated literature search using the same methodology was conducted in November 2016. The search strategy for this literature search is presented in table 1.

Titles were screened (YCH-T) for appropriateness. Two authors (YCH-T, NMP) independently reviewed abstracts to further eliminate studies not meeting the selection
Table 1 Search criteria

Outcome terms $\dagger$ Health status OR successful ag*ing OR healthy $\mathrm{ag}^{\star}$ ing OR positive $\mathrm{ag}^{\star}$ ing OR ag*ing well OR longevity

\section{AND}

Factor terms Factor* OR predict* OR indicator ${ }^{*}$ OR determinant

$\begin{aligned} \text { Filters } & \text { Published between January } 2000 \text { and } \\ & \text { November } 2016 \\ & \text { Human subjects } \\ & \text { English language } \\ & \text { Population aged } 65 \text { years or older }\end{aligned}$

*Indicates the term is truncated or has spelling variation. †These terms were adopted in search criteria since this nomenclature dominates the literature describing a multidimensional composite measure of health status in older age. $^{10}$

criteria presented in table 2 . The full text of all remaining articles was retrieved and the decision to include in the review was made by two authors (YCH-T, NMP) in consultation with third author (REH) where doubt existed. In addition, reference lists of included articles were searched to identify other studies meeting the inclusion criteria.

\section{Data extraction}

Two authors (YCH-T and NMP) independently extracted the data on study population, study design, measures of health status, all modifiable social, personal, economic and environmental factors, analyses and results using a standardised spreadsheet. Data were compared and agreement on study variables reached by consensus. Study characteristics are reported in table 3. Measurement of the outcome, health status as well as prevalence in the study population was documented. Factors that positively influenced (or were protective of) health status were classified under personal, social, economic and environmental domains.

\section{Data synthesis and analysis}

Data were synthesised and reported according to the preferred reporting items for systematic reviews and meta-analyses (PRISMA) statement. ${ }^{12}$ Due to the heterogeneity of study populations, outcome and predictor measures, a meta-analysis was not possible.

\section{Study quality}

Studies were evaluated for methodological quality using an appraisal instrument, previously proven to be a valid and reliable tool for use in epidemiological studies. ${ }^{13}$ Twenty questions relevant to comparative and observational studies were used from this instrument; scores for each question ranged from 2 to 0 , depending on whether the question was fully, partially or not addressed. An average score was calculated for each study, which could then be classified into low-quality, medium-quality or high-quality categories. The criteria for quality assessment 


\begin{tabular}{|c|c|c|}
\hline Criteria & Included & Excluded \\
\hline Publication type & $\begin{array}{l}\text { Published in peer-reviewed scientific journals } \\
\text { Reporting original research results } \\
\text { Written in English }\end{array}$ & $\begin{array}{l}\text { Reviews, book chapters, editorials, } \\
\text { dissertations, theses and conference abstracts } \\
\text { 'Grey' literature }\end{array}$ \\
\hline Study design & $\begin{array}{l}\text { Observational studies with a primary aim to } \\
\text { measure associations between key determinants } \\
\text { and health in older age } \\
\text { Quantitative studies }\end{array}$ & $\begin{array}{l}\text { Qualitative studies } \\
\text { Studies evaluating models for healthy ageing }\end{array}$ \\
\hline Population & $\begin{array}{l}\text { Mean age at baseline } \geq 65 \text { years } \\
\text { Community dwelling }\end{array}$ & $\begin{array}{l}\text { Mean age at baseline }<65 \text { years } \\
\text { Hospitalised, residing in long-term care or } \\
\text { assisted living communities }\end{array}$ \\
\hline $\begin{array}{l}\text { Study factor } \\
\text { domains }\end{array}$ & $\begin{array}{l}\text { Personal } \\
\text { Social } \\
\text { Economic } \\
\text { Environmental }\end{array}$ & $\begin{array}{l}\text { Behavioural or lifestyle factors * } \\
\text { Factors which were part of multidomain } \\
\text { outcome measure }\end{array}$ \\
\hline $\begin{array}{l}\text { Outcome } \\
\text { measure }\end{array}$ & $\begin{array}{l}\text { Health status to include a composite measure } \\
\text { across multidomains of physical, mental and social } \\
\text { well-being }\end{array}$ & $\begin{array}{l}\text { Health measured as a single item question, for } \\
\text { example, self-reported health or life satisfaction }\end{array}$ \\
\hline
\end{tabular}

${ }^{*}$ Not included in this review as these factors have been a focus of a previous review. ${ }^{10}$

and the number of studies scoring a minimum of 1 point for each assessment item is included in Supplementary data 1 . Study quality was independently assessed by two authors (YCH-T, NMP) based on the instrument guidelines. $^{13}$

\section{Review quality}

A PRISMA 2009 checklist for this review is included in Supplementary data 2. This review is registered with prospective register of systematic reviews study ID: CRD42016035286.

\section{RESULTS}

The search of online databases in October 2014 identified 2819 publications. Following the exclusion of duplicates (from two or more databases) and the screening of titles and abstracts, 226 articles proceeded to full-text screening. Of these, 204 failed to meet the specified selection criteria (table 2), resulting in 22 eligible articles. Five articles were added following screening of references cited in eligible articles, taking the total number to 27. An updated literature search identified an additional three articles, as well as one article from searching the reference lists of these articles. Seven articles were excluded following the decision to remove papers reporting on factors associated with a negative health outcome (such as frailty). The final number of articles included in this review is 23. Figure 1 displays the flow diagram for selection of eligible articles for inclusion in the analysis.

\section{Study characteristics}

Study characteristics are reported in table 3. Publication dates of the 23 selected articles ranged from 2001 to 2016, analysing data from populations in the USA, ${ }^{14-17}$
Canada, ${ }^{1819}$ Asia, ${ }^{20-27}$ Europe,${ }^{28-30}$ Australia, ${ }^{31-33}$ Mexico, ${ }^{34}$ South America ${ }^{35}$ and Africa. ${ }^{36}$ Studies included 22 different population cohorts, with sample sizes ranging from 67 to 10048 participants and mean age between 70 and 87 years. Most studies included both males and females, in which the proportion of females varied from $39 \%$ to $82 \%$. Two were male only. ${ }^{14}{ }^{17}$ Cross-sectional analysis was used in 16 studies, with the remaining 7 studies $^{14171925293236}$ using baseline data to predict subsequent health status.

\section{Health status measures}

All but one article investigated factors in relation to successful or healthy ageing. Studies used different definitions, with the majority basing health measures on the model of Rowe and Kahn, ${ }^{37}$ who defined successful ageing as the avoidance of disease and disability, the maintenance of high physical and cognitive function and sustained engagement in social and productive activities. One article ${ }^{25}$ measured health status using a health index, which, similar to the healthy ageing model, assessed physical and cognitive function, psychological well-being and subjective health to provide a composite measure. The prevalence of successful/healthy ageing ranged from $1 \%$ in the Hong Kong sample, meeting criteria for high functioning in all four domains (physical, affective and cognitive functioning and productive involvement), ${ }^{21}$ to $81 \%$ in a community sample from Singapore,${ }^{26}$ who met criteria on physical, mental and social health.

\section{Determinants of health status}

Personal

A total of 12 articles investigated personal factors as determinants of health status. ${ }^{1417182021242729-313336}$ Personal 


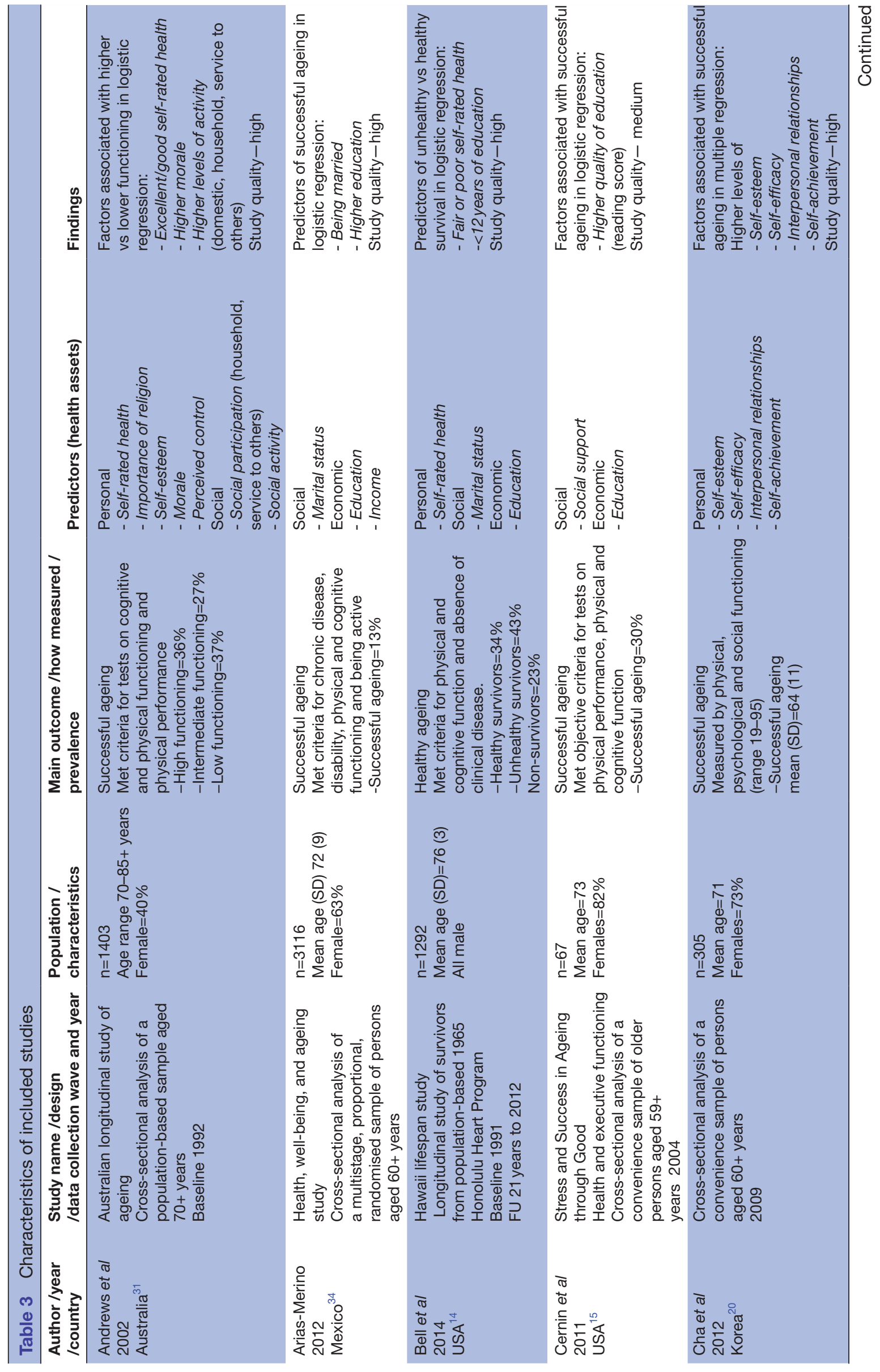

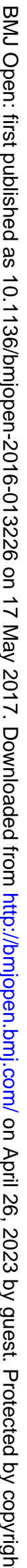




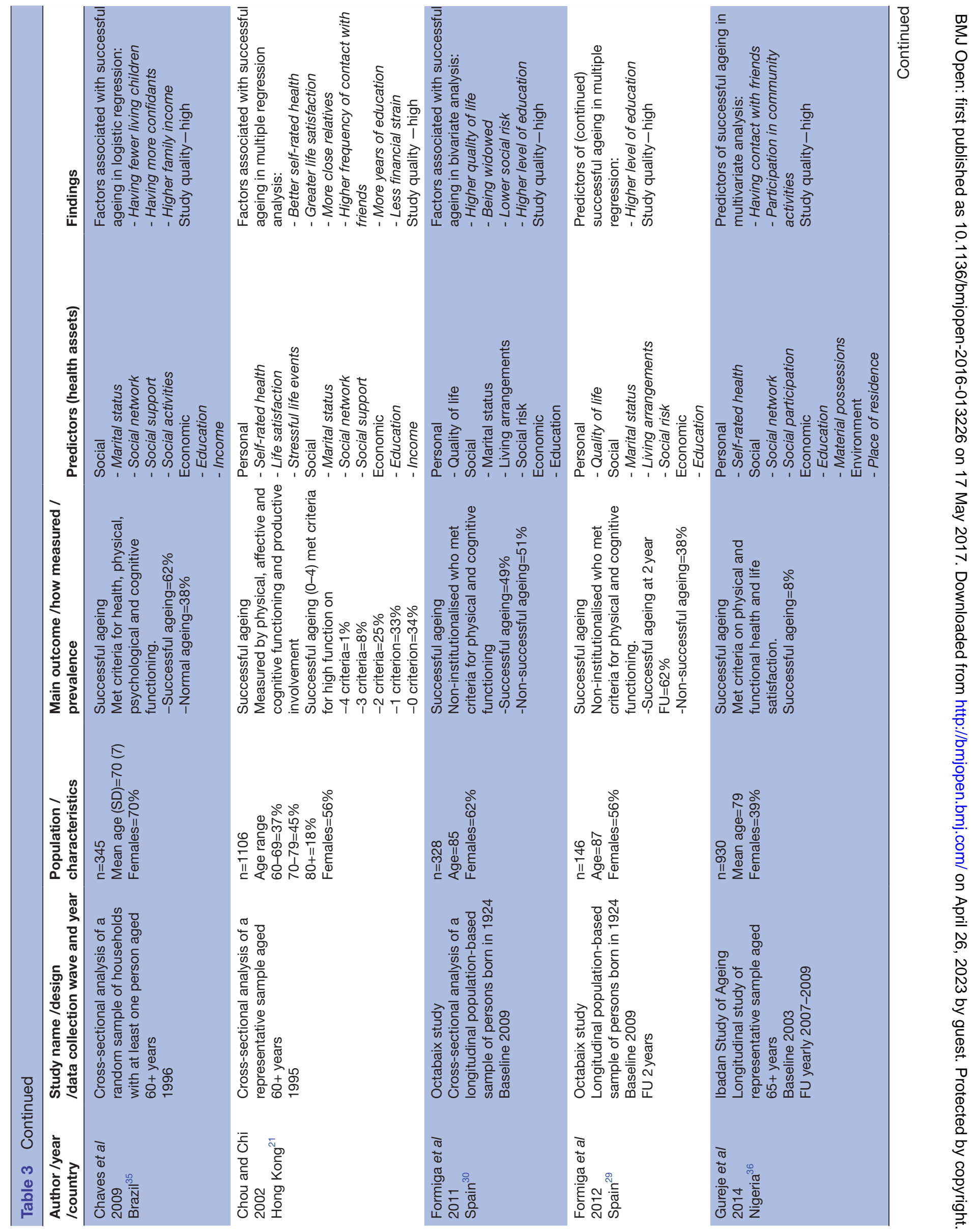




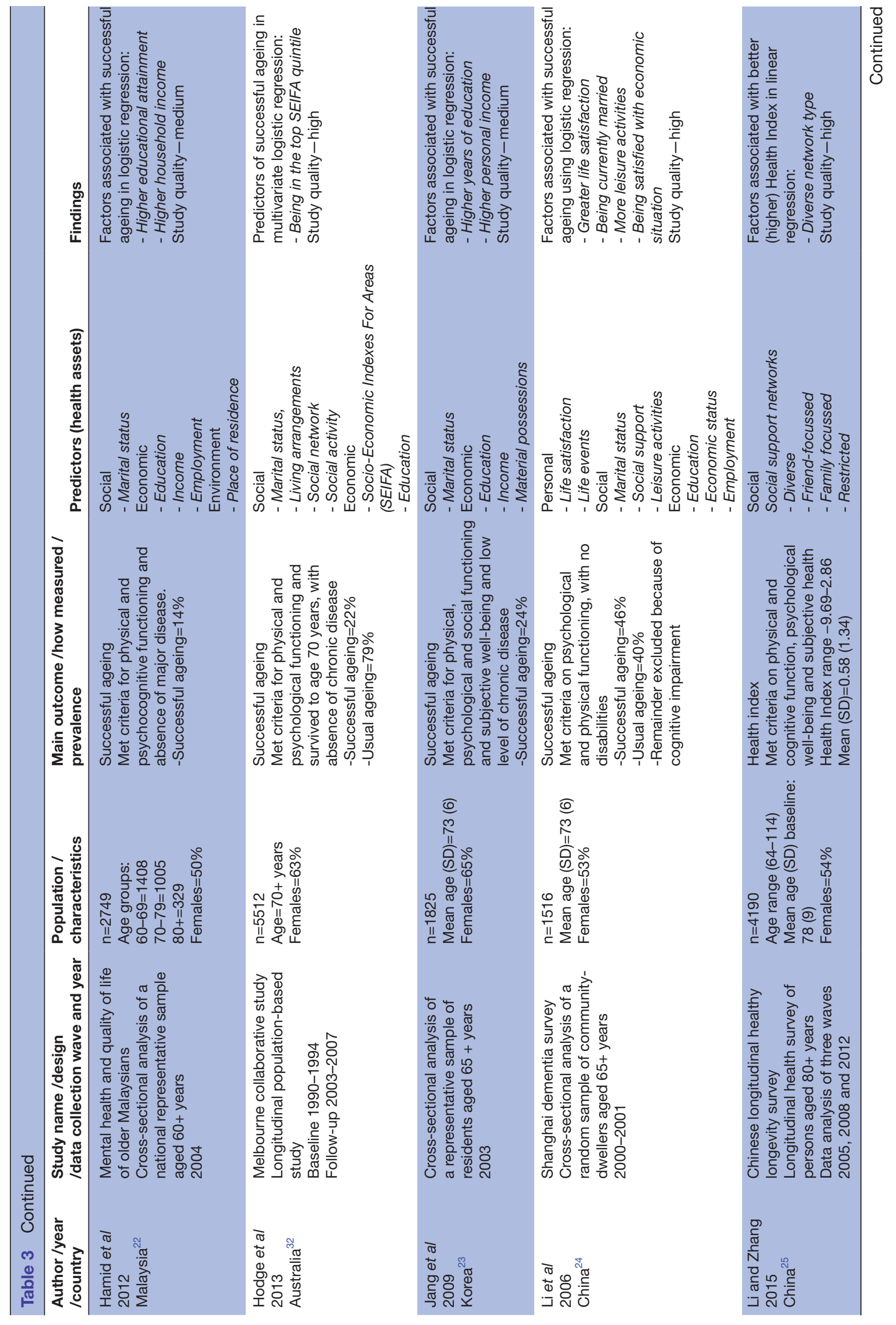

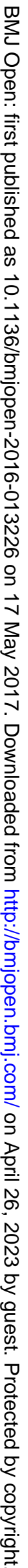




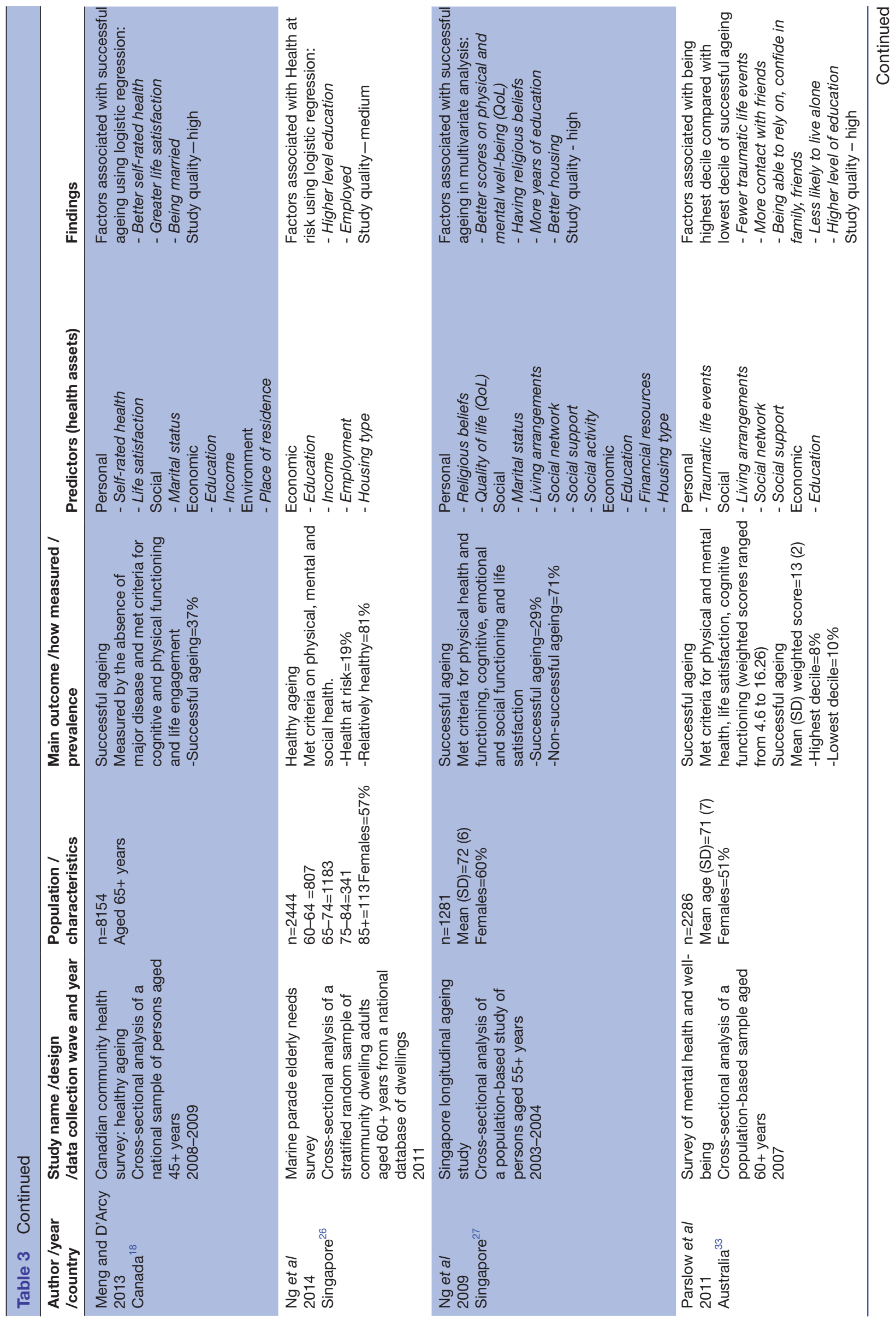




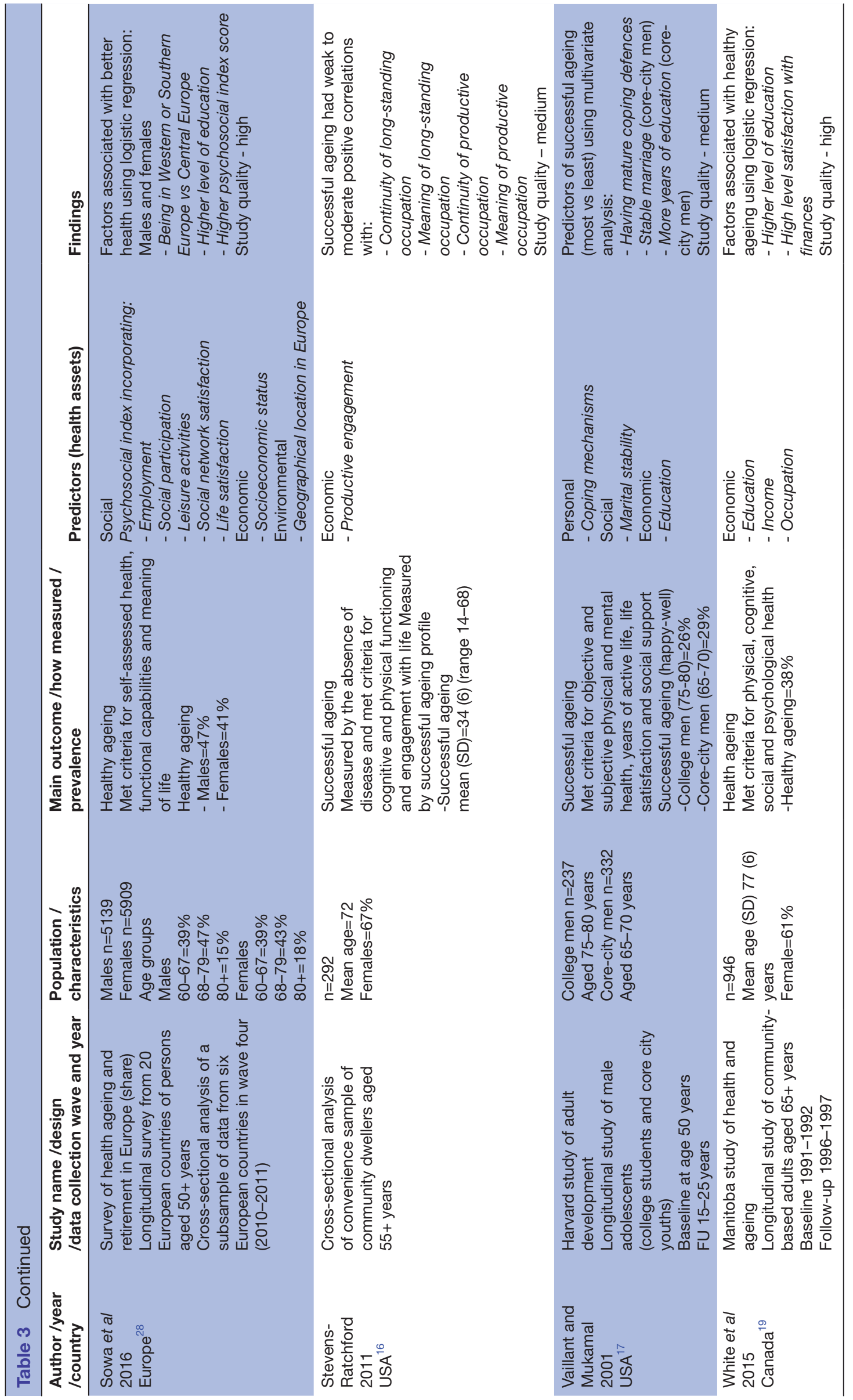




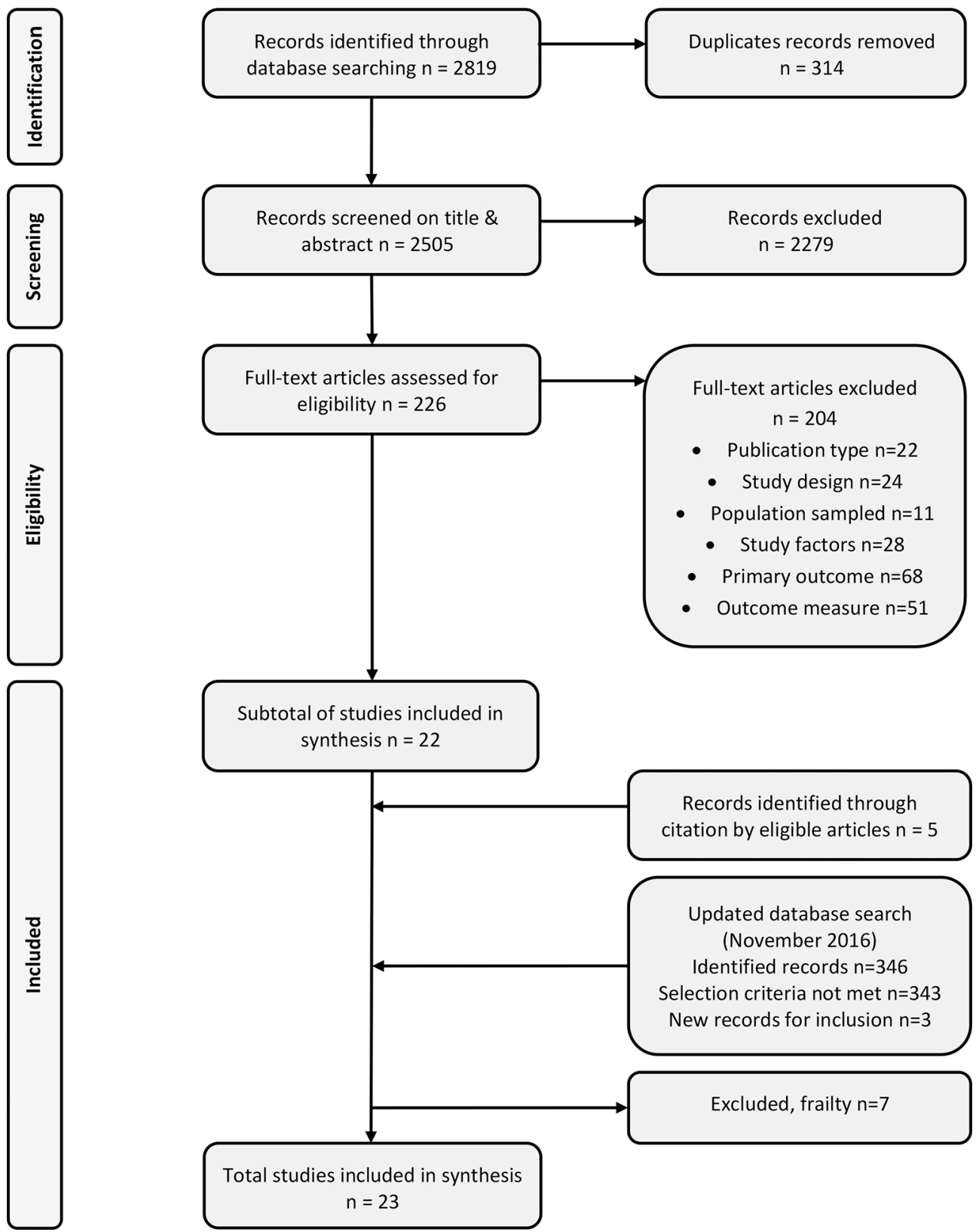

Figure 1 Flow diagram of article selection.

factors incorporate a wide range of attitudes, perceptions and internal resources that relate to health and wellbeing.

Self-rated health, measured on a scale from poor to excellent, was investigated in five studies. ${ }^{14} 18213136 \mathrm{~A}$ significant relationship between self-reported health and successful ageing was reported in all but one study, ${ }^{36}$ suggesting those who perceived their health as good to excellent were more likely to age successfully than those who perceived their health as fair to poor.

Well-beingwas investigated in ninestudies. ${ }^{17182021242729-31}$ Higher levels of self-esteem, self-achievement, self-efficacy, interpersonal relationships ${ }^{20}$ and religious beliefs ${ }^{27}$ were found to be associated with successful ageing, while a higher morale was associated with higher functioning. ${ }^{31}$ Successful agers expressed greater life satisfaction ${ }^{18} 2124$ and a higher quality of life $\mathrm{e}^{27} 30$ in cross-sectional analysis. However, quality of life was no longer a predictor of continued successful ageing in the Octabaix study in longitudinal follow-up. ${ }^{29}$ Having mature coping mechanisms ${ }^{17}$ and fewer traumatic life events ${ }^{33}$ were also found to be associated with successful ageing.

\section{Social}

A total of 19 articles investigated social factors as determinants of health status. ${ }^{14} 151718$ 21-25 27-36 Two studies screened multiple factors to create a composite measure of social risk. Formiga et $a t^{29}{ }^{30}$ used the Gijon scale to assess family and economic situation, housing, relationships and social support as a composite measure of social risk. Data were collected from this Spanish sample at both baseline and 2-year follow-up. A lower score on the 
social risk scale was associated with successful ageing in cross-sectional analysis ${ }^{30}$; however, this association was no longer significant in longitudinal follow-up. ${ }^{29}$ Sowa $e t$ $a l^{28}$ used a psychosocial index based on a combination of social and personal factors, including employment, social participation, leisure activities and satisfaction with social network, in a subsample of the European SHARE data. A higher score on the psychological index was associated with better health in cross-sectional analysis in both the male and female samples.

Marital status and living arrangements were investigated in 13 articles. ${ }^{14} 1821-2427293032-35$ Being married, or not living alone, were positively associated with successful ageing. ${ }^{18} 243334$ In contrast, the Octabaix study found being widowed was associated with successful ageing at baseline, 85 years of age, but not at follow-up 2 years later. $^{29}{ }^{30}$ A longitudinal study, of two cohorts of adolescent boys (college students and city youth) in the USA, investigated marriage stability and its ability to predict health status in later life. ${ }^{17}$ For the city cohort, having a stable marriage in midlife was a predictor for successful ageing in later life. This factor did not influence health status in the college cohort.

Social network, commonly measured by the number and frequency of contact with family, friends and neighbours was investigated in seven studies. ${ }^{21} 252732333536$ Having a wide social network ${ }^{21}$ and close contact with friends ${ }^{21} 3336$ was found to support successful ageing in all but one ${ }^{35}$ of these studies. Li and Zhang ${ }^{25}$ investigated a range of social support network types and their effect on health status in a Chinese population, aged 80 years and over. Those who had a diverse network, including contact with family and friends, as well as participation in social activities, had better health than those with either a restricted, friend or family-only focused network type. However, a South American study, ${ }^{35}$ using cross-sectional analysis, found having fewer living children was associated with successful ageing in their largely female $(70 \%)$ sample.

Social support, measured in terms of emotional or instrumental support was investigated in five study cohorts; three of which found having confidants and support from family and friends were positively associated with successful ageing. ${ }^{21} 3335$ In an Australian sample of persons aged 70 years and over, ${ }^{31}$ providing support to others in cross-sectional analysis was significantly associated with higher level functioning.

Engagement in social activities was investigated in six studies. ${ }^{24} 2731323536$ Participation in community-leisure activities was found to be associated with successful ageing in two study cohorts. ${ }^{24} 36$ Finally, participation in domestic and household activities was found to be a protective factor in successful ageing in a sample of older Australians. ${ }^{31}$

\section{Economic}

A total of 20 studies investigated economic factors as determinants of health status. ${ }^{14-19}$ 21-24 26-30 ${ }^{32-36}$ All studies included education as an economic indicator in their investigations, with level of attainment and years of study the most common measures of education. Thirteen of these studies found more years, or a higher level of education was associated with, or predictive of successful ageing in cross-sectional ${ }^{21-2326-28303334}$ as well as longitudinal $^{14171929}$ data analysis. One study focused on the quality of education derived from a reading score, ${ }^{15}$ showing that a higher quality of education was associated with successful ageing.

Income was investigated in eight studies. ${ }^{18} 19$ 21-23 263435 In cross-sectional analyses, having higher personal, ${ }^{23}$ or household ${ }^{22} 35$ income was associated with successful ageing. Financial strain was investigated in three studies, cross-sectional $^{2124}$ and longitudinal analysis ${ }^{19}$ of these data found those reporting that their financial resources were adequate for their needs were more likely to age successfully than those experiencing financial strain.

Occupation class or employment status was investigated in four articles. ${ }^{16} 192224$ Of these, one study ${ }^{26}$ found being employed was associated with better health, and a second ${ }^{16}$ found a weak-to-moderate correlation between continuity and meaning of occupation and successful ageing.

The influence of housing type $\mathrm{e}^{26}$ and material possessions $^{23}{ }^{36}$ on successful ageing was investigated in four studies. One study ${ }^{27}$ found better housing was associated with successful ageing in cross-sectional analysis.

A composite measure of socioeconomic status was investigated in data from the Melbourne collaborative study. ${ }^{32}$ Based on census data, the Socio-Economic Index for Areas (SEIFA) is an index of relative socioeconomic disadvantage, measuring, at an area level, factors such as income, education and occupational status. Longitudinal analysis found, being in the top SEIFA quintile was a predictor of successful ageing.

\section{Environmental}

Environmental factors, including geographical location ${ }^{28}$ and place of residence, ${ }^{18} 2236$ were investigated in relation to successful ageing in four studies. The latter three studies examined the effect of urban versus rural locality on successful ageing and found no significant relationship. However, those residing in Western or Southern Europe were more likely to be in the healthy ageing group, compared with those in Central Europe.

\section{Study quality}

Using the modified Epidemiological Appraisal Instrument, scores for assessment of methodological quality ranged from 14 to 36 , out of a possible 40 points, with an average score of 27.8 points. Studies were classified into low quality (0-13), medium quality (14-27) or high quality (28-40) categories, determined by their final score. Study quality results are reported in table 3. The assessment criteria that were most poorly reported by the studies included in this review were the participation rates, and the reliability and validity of the exposure variables (see Supplementary data 1 ). 


\section{DISCUSSION}

This narrative systematic review summarises the evidence for factors within personal, social, economic and environmental domains that can be termed 'health assets' of older adults. Of these, there was strong evidence from multiple high quality studies to suggest self-rated health, life satisfaction, psychological well-being, social networks, engagement in leisure and social activities, education and financial resources are associated with health status in community dwelling older populations.

Although the review included studies from a diverse range of countries in the high-income and middle-income world, cross-national comparisons of factors influencing ageing well were not possible because of differences in population sample characteristics, health status and study factor measures. The prevalence of successful ageing covered a wide range from $1 \%$ to $81 \%$. The one study incorporating cross-country comparisons found the differences in healthy ageing could be attributed to the prevalence of chronic conditions in Central-Eastern Europe as opposed to Western or Southern Europe. Education was the most commonly studied factor in this review with strong evidence cross-nationally that a higher level of education is widely associated with positive health in older age. This study was unable to identify any specific trends in health assets that were attributable to geographical diversity. However, we recognise that differences in access to resources and healthcare services can vary significantly by geographical location and consequently impact health.

The majority of studies included in this review measured factors individually, even though their effects are often interdependent and additive. ${ }^{38} \mathrm{~A}$ small number of studies, however, used composite measures including a multidomain measure of social risk, ${ }^{29}{ }^{30}$ and single domain multifactor measure of socioeconomic status. ${ }^{32}$ A multidomain summative measure of protective factors was investigated in older adults in Beijing. ${ }^{38}$ This study reported that for each accrued protective factor, the risk of health decline and death was reduced by $13 \%-25 \%$. These data suggest that the more protective factors the individual possesses, the more the risk of poor health is reduced and the greater the opportunity for recovery. The rationale underpinning the study of 'health assets' is similar to that of 'health deficits'; both measure an accumulation of factors across multiple domains that predict health status. While an accumulation of deficits predicts ill health, an accumulation of health assets may mitigate risk and promote good health. This highlights potential for a 'health assets' tool to evaluate cumulative factors known to positively influence health and well-being. Such a tool could be useful in epidemiological studies to examine why individuals have different health outcomes depending on their level of health assets.

A person's health and well-being has many facets, resulting from a complex interplay between factors within multiple domains. ${ }^{2}$ Such factors are highly influenced by cultural norms, gender-specific roles ${ }^{3}$ and the resources and policies of the wider society. ${ }^{39}$ The modifiability of these factors therefore can be highly dependent on the individual and the context in which they live. While some factors are seemingly immutable at the individual level, population health policies to reduce poverty provide social support, connection to culture and equitable access to healthcare can protect against the effects of living in disadvantaged circumstances. Other factors under personal control, for example, engagement in leisure and social activities, are more amenable to interventional programmes and policies. ${ }^{39}$ Furthermore, enabling people to develop and maintain varied social networks and participation in social and recreational activities may help them on a social level and can have a positive impact in other domains including maintaining independence, life satisfaction, well-being and physical and mental health.

The mechanism through which health assets can influence health may be direct or indirect. For example, those on very low incomes may lack resources and access to adequate housing, safe environments and healthcare, which can impact negatively on health. Financial and life stressors, as well as lack of resources, social support and connectedness can contribute directly to poorer physiological health (eg, increased risk of high blood pressure, immune and circulatory complications) or indirectly, through less healthy coping skills and behaviours (eg, excessive alcohol consumption or substance abuse). Although self-rated health is a consistent indicator of objective health and a robust predictor of health outcomes, little is known about the mechanism by which it influences health status. ${ }^{40}$ The degree of control that people believe they possess over their personal health may increase an individual's self-rated health and lower disease burden. ${ }^{40}$

\section{Implications of findings}

Health interventions addressing personal, social, economic and environmental determinants may reduce health-related inequalities and the risk of disease late in life. ${ }^{4142}$ This review provides evidence of health assets that can be applied across the life course to promote better health and well-being into old age.

Although many health assets are already present in our lives, the individual and others around them may not necessarily be mindful or make purposeful use of them. Empowering people to recognise and build on their potential health assets may help protect and promote health status. An asset-based approach to health promotion exposes and values the skills, knowledge, connections and the potential of the individual and those around them. ${ }^{43}$ The aim of this approach is to strike a balance between meeting the needs and nurturing the strengths and resources of the individual and community. Demographic changes in global ageing means that more people will require help and support. This asset-based approach is an ideal opportunity for government health bodies and their partners to respond to these challenges. 


\section{Strengths and limitations of this study}

This review has evaluated an extensive range of health assets, highlighting the strongest evidence for factors that positively influence health in older age.

Methodological differences in study design, follow-up periods, population samples and the way health assets and outcomes were measured by the studies included in this review precluded the pooling of results for meta-analysis. Including only papers published in English is acknowledged as a limitation, affecting cross-cultural comparisons and ability to generalise results to non-English-speaking countries.

Cross-sectional analysis in the majority of studies did not allow for investigation of causality, while longitudinal analysis was largely unidirectional, with study factors such as better self-rated health, social network support and higher educational attainment predicting subsequent successful ageing. Only one study ${ }^{25}$ examined bidirectional relationships, showing that social network types were predictive of subsequent health status, and that a decline in health affects social network type.

\section{CONCLUSIONS}

This systematic review summarises the evidence for health assets, thus adding to the currently limited body of literature within this field. This evidence is essential for the preparation of appropriate policies and effective health interventions.

Health assets are the individual's accessible internal or external strengths and resources; empowering people to recognise and build on their health assets may help protect and promote health status in older age. Implementing an asset-based approach to health promotion uncovers the skills, knowledge, connections and the potential of the individual and the community. This approach is an ideal opportunity for government health bodies and their partners to respond to the challenges faced by global ageing.

Factors known to influence health are often interdependent and cumulative, but the effect on health of a multidomain, composite measure of positive factors is largely unknown. This suggests potential for an instrument to measure the cumulative effect of multidomain health assets on health status in older adults.

Contributors $\mathrm{YCH}-\mathrm{T}$ assisted in the design of the study protocol and methodology, searched and screened the articles, extracted, synthesised and analysed the data and wrote the first draft of the manuscript. NMP designed the study protocol and methodology, screened, extracted, synthesised and analysed the data. REH formulated the idea for the study, assisted with the design of the study protocol. All authors contributed significantly to the preparation and revisions of the manuscript and approve the final version.

Competing interests None declared.

Provenance and peer review Not commissioned; externally peer reviewed.

Data sharing statement All data are contained in this article and no additional data are available.

Open Access This is an Open Access article distributed in accordance with the Creative Commons Attribution Non Commercial (CC BY-NC 4.0) license, which permits others to distribute, remix, adapt, build upon this work non-commercially, and license their derivative works on different terms, provided the original work is properly cited and the use is non-commercial. See: http://creativecommons.org/ licenses/by-nc/4.0/

(C) Article author(s) (or their employer(s) unless otherwise stated in the text of the article) 2017. All rights reserved. No commercial use is permitted unless otherwise expressly granted.

\section{REFERENCES}

1. World Health Organisation, National Institute on Aging. Global health and aging. 2011 http://who.int/ageing/publications/global_health. pdf?ua=1 (accessed 28 Apr 2016).

2. World Health Organisation. Active ageing: a policy framework. 2002 http://apps.who.int/iris/bitstream/10665/67215/1/WHO_NMH_NPH_ 02.8.pdf (accessed 28 Apr 2016).

3. United Nations, Department of Economic and Social Affairs, Population Division. World Population Ageing 2013. 2013 http:// www.un.org/en/development/desa/population/publications/pdf/ ageing/WorldPopulationAgeing2013.pdf (accessed 21 Jan 2017).

4. Peel N, Bartlett $\mathrm{H}$, McClure R. Healthy ageing: how is it defined and measured? Australas J Ageing 2004;23:115-9.

5. Morgan A, Ziglio E. Revitalising the evidence base for public health: an assets model. Promot Educ 2007;Suppl 2:17-22.

6. Lindström B, Eriksson M. Salutogenesis. J Epidemiol Community Health 2005;59:440-2.

7. Rotegård AK, Moore SM, Fagermoen MS, et al. Health assets: a concept analysis. Int J Nurs Stud 2010;47:513-25.

8. Seligman MEP, Peterson C, Barsky AJ, et al. Postitive health and health assets: re-analysis of longditudinal datasets. 2013 https://ppc.sas.upenn.edu/sites/ppc.sas.upenn.edu/files/ positivehealthassetspub.pdf (accessed 28 Apr 2016).

9. Gregorevic KJ, Lim WK, Peel NM, et al. Are health assets associated with improved outcomes for hospitalised older adults? A systematic review. Arch Gerontol Geriatr 2016;67:14-20.

10. Peel NM, McClure RJ, Bartlett HP. Behavioral determinants of healthy aging. Am J Prev Med 2005;28:298-304.

11. Depp CA, Jeste DV. Definitions and predictors of successful aging: a comprehensive review of larger quantitative studies. Am J Geriatr Psychiatry 2006;14:6-20.

12. Moher D, Liberati A, Tetzlaff J, et al. Preferred reporting items for systematic reviews and meta-analyses: the PRISMA statement. PLoS Med 2009;6:e1000097.

13. Genaidy AM, Lemasters GK, Lockey J, et al. An epidemiological appraisal instrument - a tool for evaluation of epidemiological studies. Ergonomics 2007;50:920-60.

14. Bell CL, Chen R, Masaki K, et al. Late-life factors associated with healthy aging in older men. J Am Geriatr Soc 2014;62:880-8.

15. Cernin PA, Lysack C, Lichtenberg PA. A comparison of selfrated and objectively measured successful aging constructs in an urban sample of African American older adults. Clin Gerontol 2011;34:89-102.

16. Stevens-Ratchford RG. Longstanding occupation: the relation of the continuity and meaning of productive occupation to Life satisfaction and successful aging. Act Adapt Aging 2011;35:131-50.

17. Vaillant GE, Mukamal K. Successful aging. Am J Psychiatry 2001;158:839-47.

18. Meng X, D'Arcy C. Successful aging in Canada: prevalence and predictors from a population-based sample of older adults. Gerontology 2014;60:65-72.

19. White CM, St John PD, Cheverie MR, et al. The role of income and occupation in the association of education with healthy aging: results from a population-based, prospective cohort study. BMC Public Health 2015;15:1181.

20. Cha NH, Seo EJ, Sok SR. Factors influencing the successful aging of older Korean adults. Contemp Nurse 2012;5:2046-66.

21. Chou KL, Chi I. Successful aging among the young-old, old-old, and oldest-old Chinese. Int J Aging Hum Dev 2002;54:1-14.

22. Hamid TA, Momtaz YA, Ibrahim R. Predictors and prevalence of successful aging among older Malaysians. Gerontology 2012;58:366-70.

23. Jang SN, Choi YJ, Kim DH. Association of socioeconomic status with successful ageing: differences in the components of successful ageing. J Biosoc Sci 2009;41:207-19.

24. Li C, Wu W, Jin H, et al. Successful aging in Shanghai, China: definition, distribution and related factors. Int Psychogeriatr 2006;18:551-63.

25. Li T, Zhang Y. Social network types and the health of older adults: exploring reciprocal associations. Soc Sci Med 2015;130:59-68. 
26. $\mathrm{Ng} \mathrm{CW}$, Luo N, Heng BH. Health status profiles in communitydwelling elderly using self-reported health indicators: a latent class analysis. Qual Life Res 2014;23:2889-98.

27. Ng TP, Broekman BF, Niti M, et al. Determinants of successful aging using a multidimensional definition among Chinese elderly in Singapore. Am J Geriatr Psychiatry 2009;17:407-16.

28. Sowa A, Tobiasz-Adamczyk B, Topór-Mądry R, et al. Predictors of healthy ageing: public health policy targets. BMC Health Serv Res 2016;16(Suppl 5):289.

29. Formiga F, Ferrer A, Alburquerque J, et al. The challenge of maintaining successful aging at 87 years old: the Octabaix study two-year follow-up. Rejuvenation Res 2012;15:584-9.

30. Formiga F, Ferrer A, Megido MJ, et al. Low co-morbidity, low levels of malnutrition, and low risk of falls in a community-dwelling sample of 85-year-olds are associated with successful aging: the Octabaix study. Rejuvenation Res 2011;14:309-14

31. Andrews G, Clark M, Luszcz M. Successful aging in the Australian Longitudinal Study of Aging: applying the MacArthur Model CrossNationally. J Soc Issues 2002;58:749-65.

32. Hodge AM, English DR, Giles GG, et al. Social connectedness and predictors of successful ageing. Maturitas 2013;75:361-6.

33. Parslow RA, Lewis VJ, Nay R. Successful aging: development and testing of a multidimensional model using data from a large sample of older australians. J Am Geriatr Soc 2011;59:2077-83.

34. Arias-Merino ED, Mendoza-Ruvalcaba NM, Arias-Merino MJ, et al. Prevalence of successful aging in the elderly in Western Mexico. Curr Gerontol Geriatr Res 2012;2012:1-6.
35. Chaves ML, Camozzato AL, Eizirik CL, et al. Predictors of normal and successful aging among urban-dwelling elderly Brazilians. J Gerontol B Psychol Sci Soc Sci 2009;64:597-602.

36. Gureje O, Oladeji BD, Abiona T, et al. Profile and determinants of successful aging in the Ibadan study of ageing. J Am Geriatr Soc 2014;62:836-42.

37. Rowe JW, Kahn RL. Successful aging. Gerontologist 1997;37:433-40.

38. Wang C, Song X, Mitnitski A, et al. Effect of health protective factors on health deficit accumulation and mortality risk in older adults in the Beijing Longitudinal Study of Aging. J Am Geriatr Soc 2014;62:821-8

39. Australian Institute of Health and Welfare. Australia's health 2010. $2010 \mathrm{http}: / /$ www.aihw.gov.au/WorkArea/DownloadAsset.aspx?id= 6442452962 (accessed 28 Apr 2016).

40. Berglund E, Lytsy P, Westerling R. The influence of locus of control on self-rated health in context of chronic disease: a structural equation modeling approach in a cross sectional study. BMC Public Health 2014;14:492.

41. Kuh D, Ben-Shlomo Y, Lynch J, et al. Life course epidemiology. J Epidemiol Community Health 2003;57:778-83.

42. World Health Organization. A life course approach to health. 2000 http://www.who.int/ageing/publications/lifecourse/alc_lifecourse training_en.pdf (accessed 28 Apr 2016).

43. Glasgow Centre for Population Health. Asset based approaches for health improvement: readressing the balance. $2011 \mathrm{http}: / / \mathrm{www}$. gcph.co.uk/assets/0000/2627/GCPH_Briefing_Paper_CS9web.pdf (accessed 21 Jan 2017). 